\title{
A new device-mediated miniprep method
}

\author{
Baryshev Mikhail ${ }^{1 *}$ (D) Merkulov Dmitrijs² and Mironov Ivan²
}

\begin{abstract}
Small-scale plasmid DNA preparation or miniprep is a fundamental technique in estimation cloning experiments and is widely used for DNA methylation analysis in epigenetic research. Current plasmid DNA minipreps use the alkali-SDS-based method in a three-solution format and require spin column-based purification steps. This procedure requires the vortexing or pipetting of pelleted bacteria by centrifugation and manual mixing of the solutions. Here, we describe a centrifuge/mixer-based instrument with the ability to perform centrifugation, vibration, and rotor oscillation in order to perform all steps of plasmid DNA isolation by device only. We found that by applying rotor oscillation-driven mixing of solutions added in the lysis and neutralization steps, homogeneous mixing was achieved within $5 \mathrm{~s}$ at a rotor oscillation amplitude of $45^{\circ}$ and oscillation frequency of $400 \pm 30 \mathrm{rpm}$, yielding the maximal quantity and quality of plasmid DNA. No increase in host chromosome presence purified by this approach occurs for highcopy-number plasmids compared to manually performed miniprep, and indeed, there is a significant decrease in the presence of the chromosomal fraction in low-copy-number plasmids. The supercoiled form of plasmid DNA purified at a rotor oscillation amplitude of $45^{\circ}$ does not turn into an open circular (OC) isoform when the plasmid is stored for 1 year at plus four degrees, in contrast to the plasmid purified with rotor oscillation amplitudes of $270^{\circ}, 180^{\circ}$ and $90^{\circ}$. The programmed time-work-efficient protocol of plasmid miniprep installed in the device gives the extreme simplicity of plasmid minipreps speeding up and facilitating the isolation of plasmid DNAs.
\end{abstract}

\section{Keypoints}

- New devise-mediated plasmid miniprep method (DM) performs all mixing steps without operator intervention.

- The DM method produces plasmid DNAs free of the dCCC form and significantly reduces the contamination with genomic DNA in the low-copy-number plasmid.

- DM miniprep plasmids are reliable templates for bisulfite PCR sequencing analysis.

Keywords: Plasmid DNA, Miniprep, Supercoiled plasmid, Plasmid DNA isolation

\section{Introduction}

Plasmid DNA minipreps are widely used procedures for recombinant plasmid purification to verify resultant constructs to fit the appropriate downstream application. To perform multiple minipreps, the cost-effective and time-efficient systems that provide researchers with the

\footnotetext{
*Correspondence: Mihails.Barisevs@rsu.lv

${ }^{1}$ Institute of Microbiology and Virology, Riga Stradins University,

Ratsupites Str 5, Riga 1067, Latvia

Full list of author information is available at the end of the article
}

appropriate quantity and quality plasmid DNA obtained with minimal manual handling are a requirement of the laboratories to compete successfully in generating results. The most commonly used procedures include alkali with SDS (Birnboim and Doly 1979), nonionic detergents (Altschuler et al. 1994) zwitterionic detergents (Chowdhury and Akaike 2005), phenol, and phenol/chloroform extraction with $\mathrm{MgCl}_{2}$ addition (Cheng et al. 2004; $\mathrm{He}$ et al. 1990; Kovalenko et al. 1994). Several approaches using the colony grown on plate agar as starting material for plasmid minipreps instead of liquid bacterial culture
Springer Open

(c) The Author(s) 2022. Open Access This article is licensed under a Creative Commons Attribution 4.0 International License, which permits use, sharing, adaptation, distribution and reproduction in any medium or format, as long as you give appropriate credit to the original author(s) and the source, provide a link to the Creative Commons licence, and indicate if changes were made. The images or other third party material in this article are included in the article's Creative Commons licence, unless indicated otherwise in a credit line to the material. If material is not included in the article's Creative Commons licence and your intended use is not permitted by statutory regulation or exceeds the permitted use, you will need to obtain permission directly from the copyright holder. To view a copy of this licence, visit http://creativecommons.org/licenses/by/4.0/. 
have been described (Sato et al. 2012, 2014). The standard alkali SDS column-based method of minipreps relies on the irreversible denaturation of bacterial genomic DNA under high $\mathrm{pH}$ and the ability of plasmid DNA to reassociate upon subsequent neutralization. While chromosomal DNA along with some RNA, protein, and cell debris remains in the precipitate, the plasmids are in solution (Jo et al. 1991). Subsequently, other contaminants are removed by the addition of washing solution. The pure plasmid was then eluted in elution buffer. In various modifications, instead of columns, the plasmid may be purified by organic extraction and ethanol precipitation or differential precipitation with a polyethylene glycol/sodium chloride mixture. The final steps of all of these methods include air-drying for 10-15 min or using a vacuum desiccator.

Plasmids are known to exist in enteric cells in different conformational states: supercoiled DNA (DNA I, or covalent closed circular DNA, i.e. CCCDNA), relaxed circular DNA (DNA II, or open circular DNA, i.e. OCDNA) and linear DNA (DNA III) forms (Vinograd and Lebowitz 1966). In many cases, due to local $\mathrm{pH}$ extremes in solutions, the anchor base pairs may be lost. This treatment can then cause the plasmid to form incongruent complementary bases or cruciform loops after neutralization, resulting in an irreversibly denatured supercoiled plasmid form (DNA IV, i.e., dCCCDNA) (Diogo et al. 1999), which is an undesirable impurity in the final plasmid preparation. Some studies have been performed to remove this plasmid DNA form with arginine affinity chromatography and by combining denaturation, selective renaturation and aqueous two-phase extraction (Sousa et al. 2009; Frerix et al. 2007).

Although the column-based alkali procedure gives ready-to-use plasmid solution, the procedure demands the vortexing or pipetting of pelleted bacteria by centrifugation and the admixing of solutions manually. Here, we describe the device with an oscillation-driving mixing option for the steps of solution admixing and the ability for pellet resuspension of 12 samples simultaneously. The time-work-efficient protocol of plasmid purification provides the extreme simplicity of plasmid minipreps, reducing the operating time by $40-60 \%$ compared to a manually operating column-based approach. The plasmid DNAs obtained by using the device are free of the dCCCDNA plasmid form and presented mostly by the CCCDNA form suitable for basic molecular biology applications, including sequencing and cell transfection, and can form complexes between the amphipathic TAT peptide and plasmid DNAs that can be used for gene delivery in eukaryotic cells (Saleh et al. 2010). In addition, commercially available "classical" plasmid DNA miniprep column-based alkali kits are compatible with the developed device and can be successfully applied using the installed time- work-efficient protocol.

\section{Materials and methods \\ Bacterial strain and DNA plasmids}

Invitrogen (Carlsbad, USA), supplied the XL1-blue Escherichia coli strain used for plasmid propagation in LB medium. Antibiotics were purchased from SigmaAldrich Biochemie GmbH (Hamburg, Germany). The high copy number plasmids used were pcDNA 3.1/His/ LacZ $8.6 \mathrm{~kb}$ from Invitrogen Corp. (Carlsbad, USA); pEGFP and pET30 vectors Clontech (Palo Alto, USA); and the low copy number pR322 plasmid from our lab. The pCR4-TOPO Invitrogen (Carlsbad, USA) vector was used to clone 585 and 750 bp PCR-generated fragments of PPARg2 and Oct4 gene promoter regions that were used in DNA methylation analysis by PCR bisulfite sequencing. The ZymoPURE ${ }^{\mathrm{TM}}$ II Plasmid Midiprep Kit was from Zymo Research Corp. (Irvine, USA). The QIAGEN Plasmid Midi Kit was from QIAGEN GmbH (Hilden, Germany).

\section{Standard column-based plasmid miniprep}

Standard column-based plasmid minipreps were performed according to the GeneJET Plasmid Miniprep Kit, ThermoFisher Scientific Inc. (Carlsbad, USA). (https:// tools.thermofisher.com/content/sfs/manuals/MAN00 12655_GeneJET_Plasmid_Miniprep_UG.pdf).

\section{DNA quantification}

Average concentrations of the plasmid DNA were quantified using the NanoDrop microvolume sample retention system, Thermo Fisher Scientific Inc. (Waltham, MA).

\section{Plasmid DNAs storage}

Plasmid DNAs purified by the DM and SM miniprep methods were stored in a cold room at $4-6{ }^{\circ} \mathrm{C}$.

\section{Restriction analysis and DNA sequencing}

Restriction endonucleases and T4 DNA ligase were obtained from Thermo Fisher Scientific Inc. (Carlsbad, USA), and used with the buffer stocks recommended and provided by the company.

DNA sequencing was performed using the ABI BigDye Terminator Cycle Sequencing Kit v3.1 ThermoFisher Scientific Inc. (Carlsbad, USA) according to the manufacturer's instructions on a Gene Amp 9700 PCR machine (Carlsbad, USA), and the sequences were detected on an ABI 3130XL Genetic Analyzer, Applied Biosystems (Carlsbad, USA). 


\section{Assessment of host genomic DNA in minipreps by SQ-PCR}

To obtain semiquantitative data on the presence of host genomic DNA in plasmid preparation, four $5 \times$ fold serial dilutions of host chromosome [purified with the Wizard gDNA purification kit; Promega Corp. (Madisson, USA)] in the range $0.008-1 \mathrm{ng}$ were used as the reference of host DNA amount in PCRs and compared with amplification of $50 \mathrm{ng}$ plasmid DNAs. Forward, 5'-TTCCCACGGACATGAAGACTACA-3' ${ }^{\prime}$ and reverse, $5^{\prime}$-ATCCTGCGCACCAATCAACAA-3' $E$. coli $\mathrm{K}-12$ strain-specific primers were used to amplify a $1.687 \mathrm{bp}$ fragment (Kuhnert et al. 1995). The relative intensity of the band of the genomic DNA amplified from the plasmid DNA minipreps was quantified in relation to the bands of reference diluted samples by image analysis software Science Lab Image Gauge Ver. 4.0, Fujifilm Corp. (Tokyo, Japan).

\section{Time efficiency}

The time efficiency of the device-mediated minipreps (DM) relative to the standard column-based minipreps (SM) was calculated as the ratio of the total SM and DM procedure completion times amplified by 100 . T (tot) $\mathrm{SM} / \mathrm{T}$ (tot) $\mathrm{DM} \times 100=\%$ time efficiency. Subtracting $100 \%$ we will find how much the DM minipreps more time-efficient, in percentage, compared to SM. T (tot) was considered the sum of the handling times, the time needed for sample/solution manipulation by the operator, and the technological times, which were mediated by the working equipment and incubation time according to the appropriate protocols.

\section{PC3 cell line transfection}

Prostate cancer-derived PC3 cells were cultured in RPMI-1640 culture medium supplemented with $10 \%$ fetal bovine serum [Invitrogen Corp. (Carlsbad, USA)] penicillin $(100 \mathrm{U} / \mathrm{mL})$, and streptomycin $(100 \mu \mathrm{g} / \mathrm{mL})$ [Invitrogen Corp. (Carlsbad, USA)] at $37^{\circ} \mathrm{C}$ in a $5 \% \mathrm{CO}_{2}$ atmosphere incubator. The cells were seeded in 24-well plates at a density of $1.8 \times 10^{5}$ per well one day before transfection to obtain $80 \%$ confluence. Before transfection, the growth medium was removed, and $350 \mu \mathrm{L}$ of Opti-MEM reduced serum medium [Invitrogen Corp. (Carlsbad, USA)] was added. The K $4^{\circledR}$ Transfection System consisting of the $\mathrm{K} 4{ }^{\circledR}$ Transfection Reagent and the $\mathrm{K} 4{ }^{\circledR}$ Multiplier were obtained from [Biontex Laboratories $\mathrm{GmbH}$ (München, Germany)] and used according to the company-provided protocol. The transfection efficiency was determined after $24 \mathrm{~h}$ of transfection as the percentage of green cells compared to the total cell number.

\section{Gel retardation assay}

One microgram of pcDNA 3.1/His/LacZ plasmid was premixed with various amounts of the GFP-TAT fusion protein $(0,2.5,5,7.5,10$, and $20 \mu \mathrm{g})$ in phosphatebuffered saline (PBS) to a final volume of $20 \mu \mathrm{l}$ and incubated at $37{ }^{\circ} \mathrm{C}$ for $30 \mathrm{~min}$ to form peptide/DNA complexes. GFP-TAT fusion was created by fusion PCR and cloned into an IPTG-inducible pET30 vector. The fusion protein was expressed in E. coli, and pure protein was subsequently purified by Ni-NTA chromatography. These complexes were analyzed by electrophoresis on a $1 \%$ agarose gel in TAE buffer, followed by staining with ethidium bromide.

\section{Results}

\section{Instrument for devise-mediated miniprep}

The instrument for device-mediated minipreps was created on the basis of an ELMI centrifuge/mixer and has a capacity of up to 12 samples (Fig. 1A). In this study, a special two-part rotor was designed and constructed to perform all steps of the miniprep with the constructed device only (Fig. 1B). The upper part of the rotor comprises the angle disk with openings, while the lower part is the disk with specially shaped openings whose axes lie in one plane with the openings of the upper part of the rotor. This feature of the rotor drives concussion of tubes in the openings of the lower part rotor under rotor vibration, which, in turn, results in quick resuspension of bacterial pellets. The introduced option of back-and-forth rotor movement performs the oscillation-driving mixing of solutions added without tube inversion.

\section{Plasmid yield by the DM miniprep depends on oscillation-driving mixing parameters and exceeds that by the SM method}

The mixing of miscible fluids of different densities must be done within the miniprep procedure. Mixing, as a degree of homogeneity of two or more liquids, plays a pivotal role in the quality and quantity of the final product. In miniprep, it is conventionally carried out by tube inversion. This step is operator-dependent, and some variability in the homogeneity of mixing might occur, which may have an effect on the yield and quality of plasmid DNA. Unlike commonly used $1.5 \mathrm{ml}$ tubes, a $2 \mathrm{ml}$ tube has a cylinder-like geometry and suite to be used in rotor oscillation-driving mixing and will promote an increase in plasmid yield.

We introduced oscillation-driven mixing to avoid manual operation and to make the process of plasmid DNA isolation 'semi-automated. The oscillating motion of the rotor provides oppositely vectored acceleration 


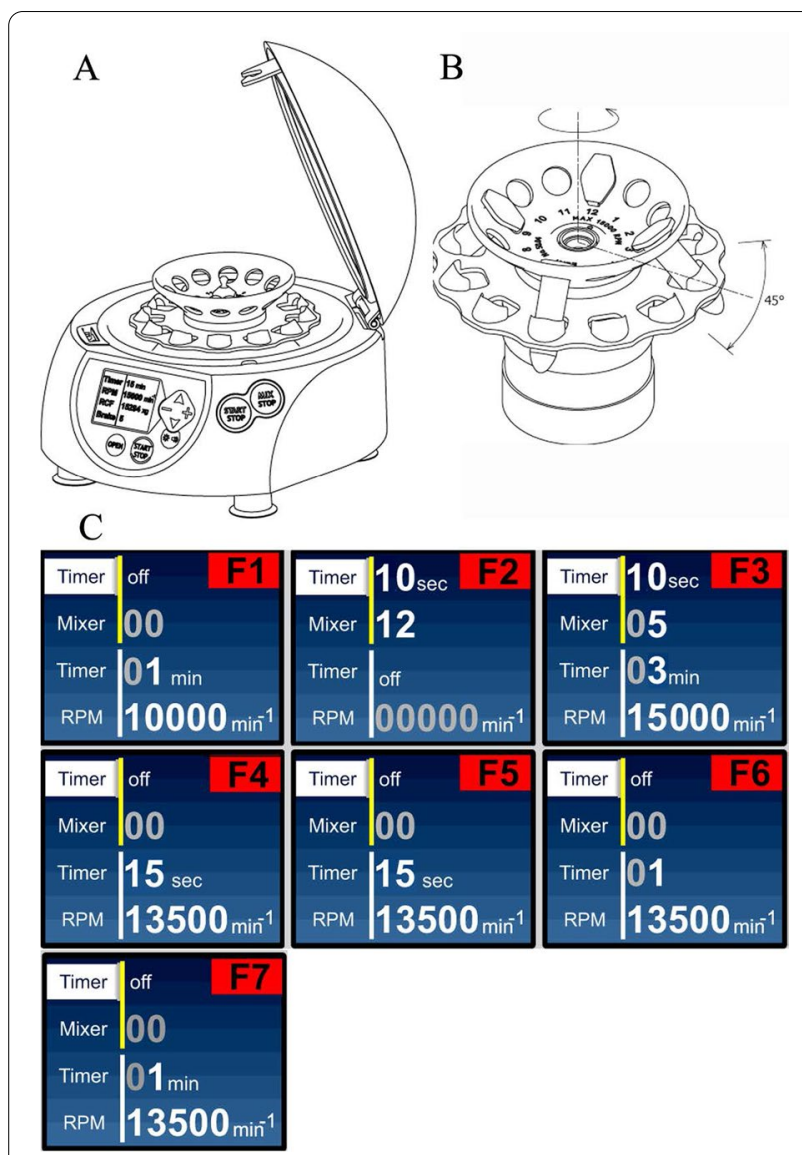

Fig. 1 Schematic view of a miniprep assisting instrument assembly. A Centrifuge/mixer-based apparatus. B Two-part rotor. C F1-F7steps of the DM miniprep protocol. Pre-set parameters for each step of device-mediated miniprep showing the different settings of parameters such as time and speed of centrifugation (F1, F3-F7), time and intensity of vibration for pellet resuspension (F2) and oscillation frequency of rotor motion at amplitude $45^{\circ}$ for liquid mixing (F3). $\mathrm{F} 3$ is a step that combines the mixing of the solution by oscillation of the rotor with an oscillation frequency of $400 \mathrm{rpm}$ (Mixer 5) and an amplitude of $45^{\circ}$ for $10 \mathrm{~s}$, after which the device automatically starts centrifugation. Amplitude of $45^{\circ}$ for liquid mixing is a constant parameter in the DM miniprep

forces, which are automatically imposed on the constituent of the tube to mix the solutions. To ensure proper mixing, the tube is periodically oscillated in opposite directions so that the various formed components will gravitate first toward one end of the tube wall and then toward the opposite end of the tube wall. We compared the effect of oscillation-driven mixing at various amplitudes and frequencies on plasmid DNA yield (Fig. 2A-D) and found that the process of mixing is time-, oscillation amplitude- and oscillation frequency-dependent and affects plasmid quality and yield. Having evaluated parameters affecting plasmid yield by this approach, we found that a combination of oscillation amplitude $45^{\circ}$ and oscillation frequency $400 \mathrm{rpm}$ ensures the homogenous mixing of the admixed solutions during minipreps in 5-10 s, yielding the amount of plasmid DNA exceeding that of the manually isolated plasmid DNA (Fig. 2A, C, D). In addition to a higher plasmid yield, an improvement in the quality of the plasmid was also observed: the pEGFP plasmid was free of the dCCCDNA plasmid form (Fig. 3A, C). It is interesting to note that the plasmid yield at a rotor oscillation amplitude of $90^{\circ}$ is less than that obtained at $270^{\circ}$ and $180^{\circ}$. Furthermore, the plasmid yield at these three amplitudes did not reach that obtained by the SM method even after $20 \mathrm{~s}$ of mixing (Fig. 2A, B), reflecting incomplete/improper mixing. In general, the mixing efficiency is increased upon decreasing the amplitude oscillation but not in the case of $90^{\circ}$. However, the mixing performance at an amplitude of $45^{\circ}$, which is half of $90^{\circ}$, and an oscillation frequency of $400 \mathrm{rpm}$ provides a homogenously mixed solution in 5-10 s, resulting in an excessive plasmid yield compared to the SM method (Fig. 2A, D). Moreover, plasmids purified under these conditions retained supercoiling after storage for one year at $+4^{\circ}$, in contrast to the plasmids isolated with rotor oscillation amplitudes of $270^{\circ}, 180^{\circ}$ and $90^{\circ}$ (Fig. 3B).

Of note, the low copy number plasmid prepared demonstrated the absence of visible chromosomal impurity on the gel, in contrast to the manually prepared plasmid (Fig. 3A, B). Having determined the mixing parameters giving improved miniprep, we established the DM miniprep and used it in the plasmid purification.

\section{Devise-mediated miniprep}

Miniprep consists of seven preprogrammed successive steps using the GeneJET column-based plasmid miniprep kit. The preset optimized parameters for each miniprep step are installed in the device and represent the DM miniprep protocol, as shown in Fig. 1C. Of what you need to perform miniprep is to add/remove solutions and press the start button of the following step to obtain the ready for use plasmid DNA in the final step.

F1. Spin $2 \mathrm{ml}$ of overnight grown bacterial culture. Remove the supernatant.

F2. Add $250 \mu$ l of resuspension solution. Press Start. All pellets will be resuspended.

F3. Add $250 \mu \mathrm{l}$ of lysis solution and then add $350 \mu \mathrm{l}$ of neutralization solution. Press Start. All solutions will be mixed and automatically centrifuged, removing the main impurity.

F4. The supernatant was poured into a column/tube ensemble. Press Start. Discard the flow throw.

F5. Add the washing solution. Press Start. Discard the flow throw. 


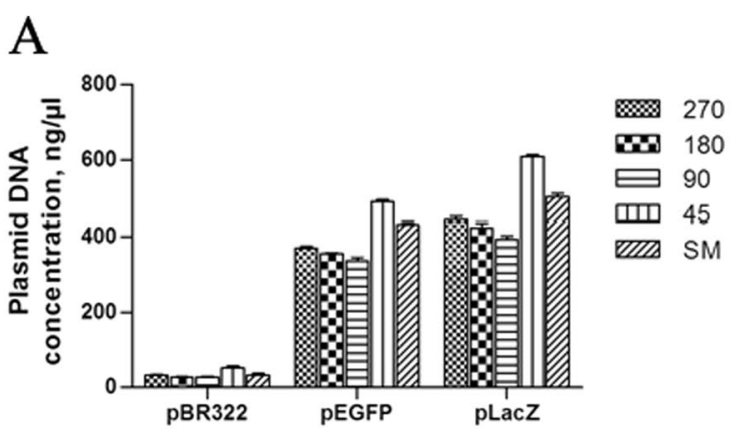

\section{$\mathrm{B}$}
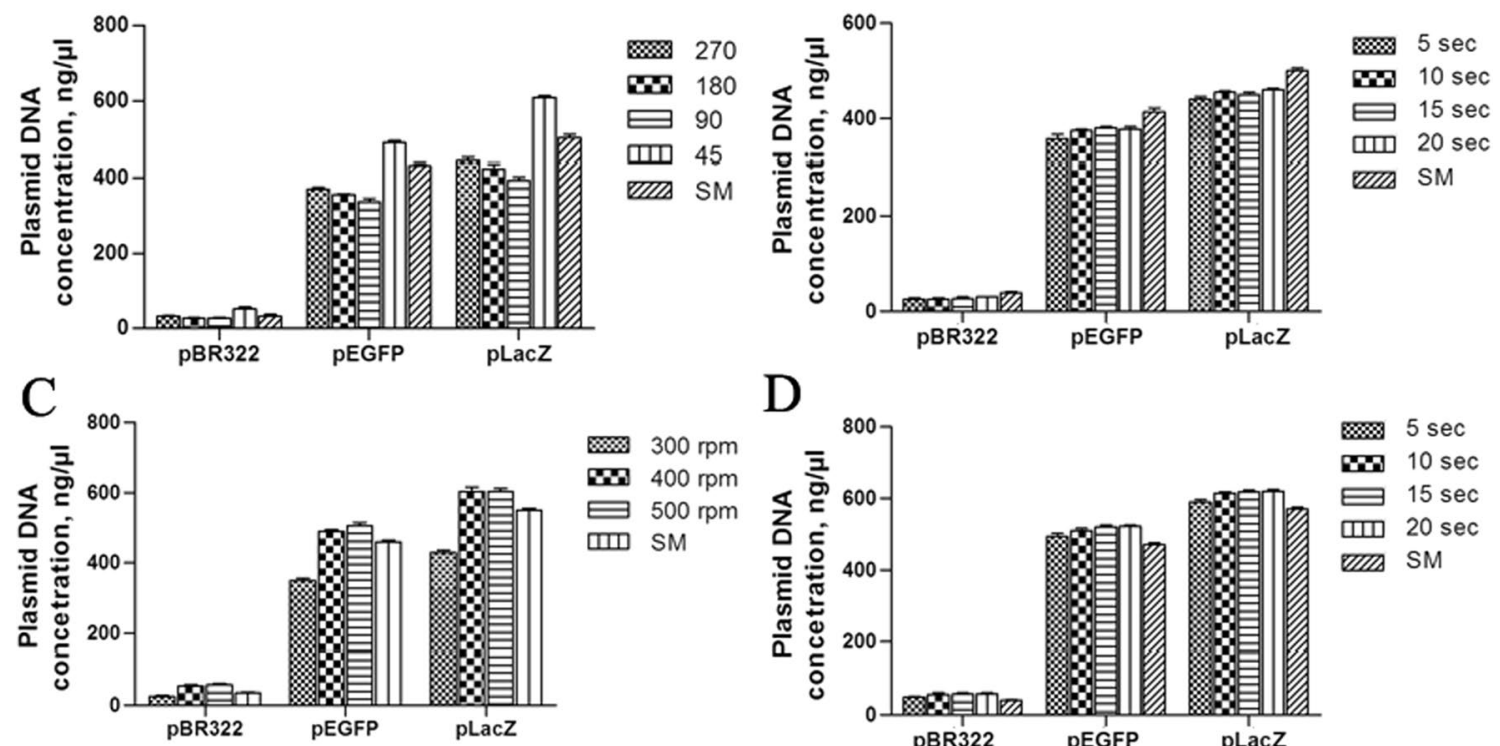

$\mathrm{D}$

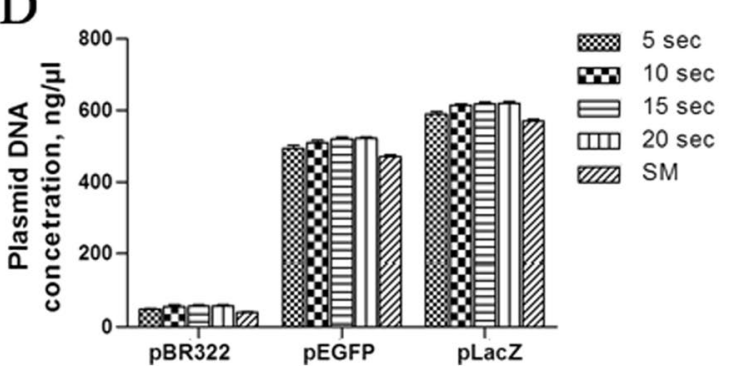

Fig. 2 Admixing efficiency of solutions performed by rotor oscillation affects the plasmid DNA yields. A Effect of oscillation amplitude of $5 \mathrm{~s}$ of oscillation-driving mixing in DM minipreps at an oscillation frequency of $400 \mathrm{rpm}$ on plasmid yields. B Effect of time oscillation driving mixing in DM minipreps at oscillation amplitude $270^{\circ}$ and oscillation frequency $400 \mathrm{rpm}$ on plasmid yields. C Effect of oscillation frequency of $5 \mathrm{~s}$ of oscillation driving mixing in DM minipreps at oscillation amplitude $45^{\circ}$ on plasmid yields. D Effect of time oscillation driving mixing at oscillation amplitude $45^{\circ}$ and oscillation frequency $400 \mathrm{rpm}$ in DM minipreps on plasmid yields. Assays were performed in triplicate, and error bars represent the standard deviation

F6. Place tubes into the device. Press Start. The remaining washing solution will be removed.

F7. Add $50 \mu \mathrm{l}$ of elution buffer and put the tubes into the device for $1 \mathrm{~min}$. Having increased the temperature within the device during operation will promote plasmid DNA elution. In a 1-min press Start. Eluted plasmid DNAs are ready to use.

Purified by this protocol, plasmids had OD260/280 in a range of 1.85-1.95, which indicates that the plasmids are pure enough to be tested in plasmid quality-sensitive downstream applications. The average yields of the plasmids obtained were $3 \pm 0.5 \mu \mathrm{g}$ for pBR322, $22 \pm 4 \mu \mathrm{g}$ for pEGFP, and $30 \pm 3 \mu \mathrm{g}$ for pLacZ. The pLacZ plasmid yield from $2 \mathrm{ml}$ overnight $E$. coli culture reached up to $30 \mu \mathrm{g}$, which corresponds to $3 \mathrm{mg}$ of the plasmid being isolated from $200 \mathrm{ml}$ bacterial culture by DM miniprep.

\section{Devise-mediated minipreps are time efficient compared to standard column-based plasmid minipreps}

The steps of miniprep performed by the newly developed device use the different settings of parameters such as the time and speed of centrifugation, the intensity of vibration used for pellet resuspension and the oscillation amplitude and oscillation frequency of rotor motion for liquid mixing. To minimize the miniprep time, the optimized miniprep procedure parameter settings were set up and installed in the device. Optimization ensures the completeness of the cell resuspension, bacterial lysis and solution mixing needed for miniprep performance. Figure $1 \mathrm{C}$ shows the parameter settings for each step. After the step is complete, the device automatically goes to the next step highlighting it on the screen. To evaluate the time efficiency, we compared the DM miniprep protocol versus the SM protocol. The data presented in Table 1 illustrate the time efficiency of DM minipreps. The plasmid isolation using DM minipreps saves time up to $60 \%$ upon processing 12 samples regarding the SM miniprep and requires $20 \mathrm{~min}$ to complete the procedure (Table 1 ). It should be noted that the recently published one-step miniprep method accomplishes one sample only for $26 \mathrm{~min}$, excluding the time for plasmid drying and dissolution (Lezin et al. 2011). Our DM miniprep excludes the manual handling of solution mixing and cell pellet resuspension and decreases the total time of the minipreps procedure, making plasmid isolation faster than ever. 


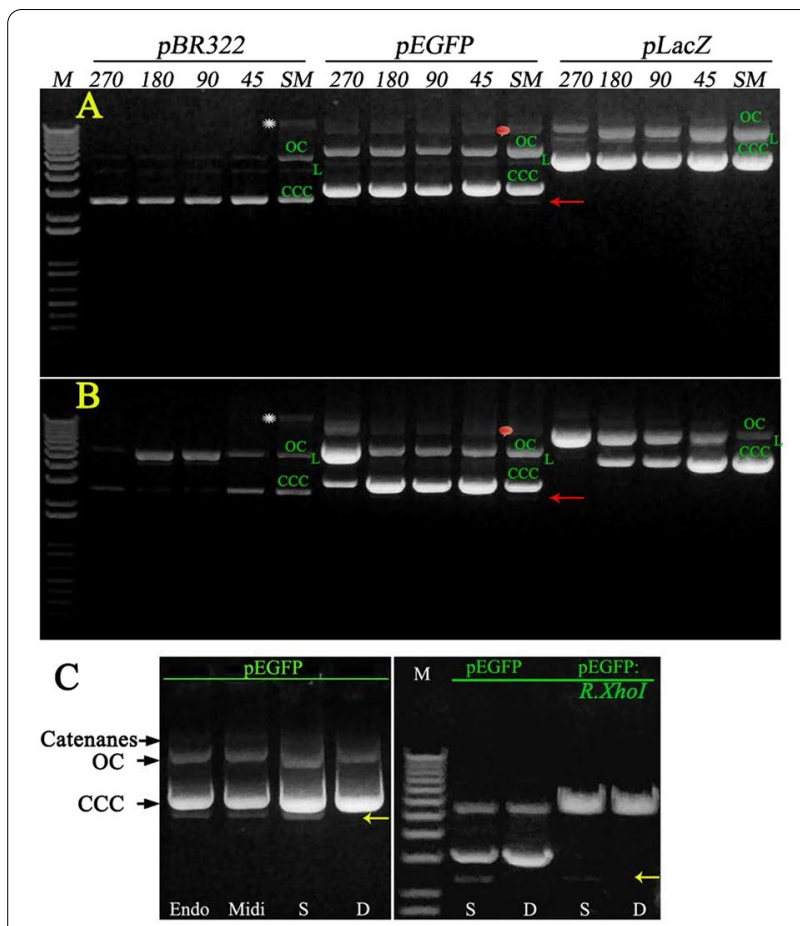

Fig. 3 DM minipreps plasmids are purer compared to SM plasmids. A Comparison of DM and SM minipreps plasmid conformational states. DM, SM miniprep plasmids were eluted in $50 \mu \mathrm{l}$ of elution buffer. $1 \mu$ l of the eluted high and $5 \mu$ low copy number plasmid were loaded in the well in a $15 \mu \mathrm{l}$ total volume. Plasmids were subjected to electrophoresis on $0.8 \%$ agarose gel. Migration of CCC, $\mathrm{OC}$ and linear $(\mathrm{L})$ forms of plasmid DNA is indicated by the green abbreviation in the corresponding band. The red pictogram shows the migration of catenated forms of pEGFP. The presence of host genomic DNA in SM-isolated pBR322 is depicted by an asterisk. B Comparison of the DNA stability of DM and SM miniprep plasmids after one year of storage at $+4^{\circ}$. DNA sample loading and legend are the same as shown in A. C Comparison of pEGFP of DM (D) and SM (S) minipreps plasmid quality and capability of cleavage by restriction endonuclease in comparison to ZymoPURE-EndoZero Midiprep (Endo) and QIAGEN Midi Kits (Midi) isolated plasmid. $1 \mu \mathrm{l}$ of pEGFP by SM and DM miniprtepped samples were digested with $1 \mu \mathrm{l}$ of Xhol at $37^{\circ} \mathrm{C}$ for $1 \mathrm{~h}$ in $15 \mu \mathrm{l}$ of the recommended buffer. The plasmid digestion products were loaded into wells and separated by electrophoresis on a $0.8 \%$ agarose gel. The $1 \mathrm{~kb}$ plus DNA ladder was electrophoresed as a DNA size marker. dCCCDNA is indicated by the arrowhead and is resistant to restriction enzyme digestion

\section{The DM plasmid is pure for restriction digestion} and sequencing, is free of the dCCC DNA plasmid form and has a lower amount of host genomic DNA

We compared the sensitivity of the plasmid DNA purified by either DM or SM minipreps in restriction enzyme digestion and sequencing reactions. Although DM and SM minipreps produced similar amounts of plasmid DNA, we observed a sustainable tendency in increasing the yield and improving the quality of plasmid DNA in the sense of plasmid topological structure and the
Table 1 Time efficiency of devise-mediated vs standard minipreps

\begin{tabular}{lllllll}
\hline & \multicolumn{2}{l}{$\begin{array}{l}\text { Devise-mediated } \\
\text { MiniPrep }\end{array}$} & & \multicolumn{3}{l}{ Standard MiniPrep } \\
\cline { 2 - 3 } $\begin{array}{l}\text { Number } \\
\text { of preps }\end{array}$ & $\begin{array}{l}\text { Total } \\
\text { time, } \\
\text { min }\end{array}$ & $\begin{array}{l}\text { Operator' } \\
\text { time }\end{array}$ & & $\begin{array}{l}\text { Total } \\
\text { time } \\
\text { min }\end{array}$ & $\begin{array}{l}\text { Operator' } \\
\text { time }\end{array}$ & $\begin{array}{l}\text { Time } \\
\text { Efficiency, } \\
\%\end{array}$ \\
\hline 2 & 12 & 4 & 17 & 5 & 42 \\
4 & 14 & 7 & & 21 & 9 & 50 \\
8 & 17 & 10 & 26 & 14 & 53 \\
12 & 20 & 13 & 32 & 20 & 60 \\
\hline
\end{tabular}

presence of chromosomal fraction among the three plasmids isolated by DM miniprep. In the pEGFP plasmid, which is often used in cell transfection experiments, we did not observe the dCCCDNA plasmid form or some chromosomal fraction visible in SM pBR322 (Fig. 3A-C).

We also found that DM miniprep plasmid DNA is a robust template in sequencing reactions of $P P A R g 2$ and Oct4 promotor region fragments of 585 and 750 bp in size, respectively, representing the bisulphite-treated inserts used in DNA methylation analysis of the genes (Additional file 1: Fig. S1, S2). All plasmids used in the analysis of DNA methylation of distal and proximal Oct4 enhancers (Baryshev et al. 2018) and the proximal PPARg2 promoter (Baryshev et al. 2022) were obtained by the DM miniprep method. We compared the presence of host genomic DNA in the plasmids obtained by DM, SM minipreps and commercial non-miniprep kits recommended for purification of high-quality plasmid DNA. From the data presented in Fig. 4A, B, it can be seen that a small fraction of the host genomic DNA in the range of $100-150$ pg per $50 \mathrm{ng}$ of plasmid is present in all purified plasmids, and the DM preparation contains less chromosomal DNA than the SM. According to densitometry analysis, the chromosomal fraction found in the plasmids was $60 \pm 5 \mathrm{pg}$ for the QIAGEN Plasmid Midi Kit, $120 \pm 10$ pg for the ZymoPURE ${ }^{\mathrm{TM}}$ II Plasmid Midiprep Kit, $105 \pm 10$ for the DM miniprep and $130 \pm 10$ for the SM miniprep (Fig. 4C, D). We further revealed that the use of DM mixing options has a great advantage for low copy number plasmid pBR322 production compared to the SM method. Any of the DM mixing parameters studied are able to produce a predominantly monomeric CCC form of the plasmid, avoiding the presence of a visible chromosomal fraction on the gel, in contrast to the SM method (Fig. 3A, B). Meanwhile, we did not observe the same effect for high-copy-number plasmids.

Thus, our results show that the hydrodynamic forces arising at a rotor oscillation frequency specified do not affect the chromosomal shearing in DM miniprep and that the content of host genomic DNA in the final DM plasmid 


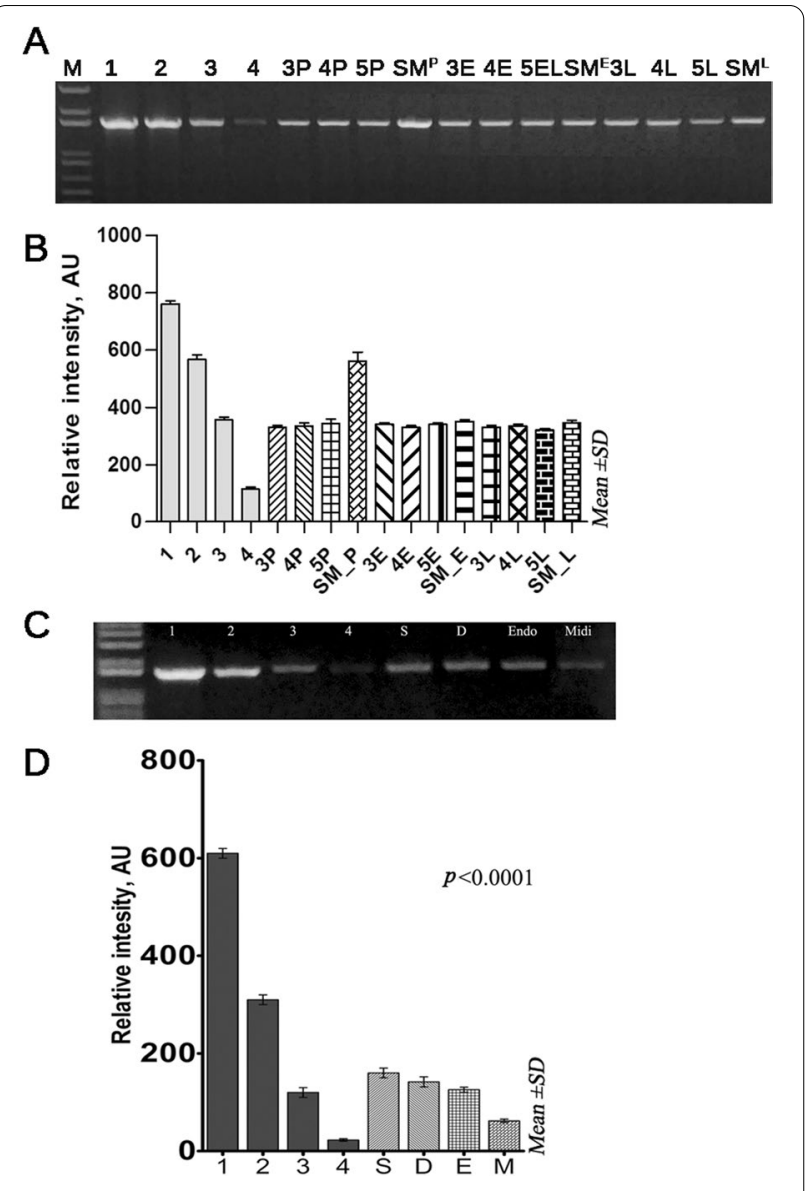

Fig. 4 DM minipreps plasmids have a lower amount of host genomic DNA. A The amount of host genomic DNA in DM and SM plasmids; lanes 1-4-reference interval of quantities' DNA for semiquantitative analysis; P, E, L-pBR322, pEGFP, LacZ plasmids purified by DM at oscillation amplitude $45^{\circ}$ and 3, 4, 5-300, 400, 500 rpm oscillation frequency; SMP ${ }^{P}, S^{E}$, SM $M^{L}$ - pBR322, pEGFP, LacZ plasmids purified by SM method. $\mathbf{B}$ Densitometry analysis of amplified products is presented. C The amount of host genomic DNA in pEGFP plasmid DNA purified either by SM (S) or DM (D) minipreps and the ZymoPURE-EndoZero (Endo) Midiprep or QIAGEN Midi kit (Midi). D Densitometry analysis of amplified products is presented. Assays were performed in triplicate, and error bars represent the standard deviation

preparation is sustainably lower than that in the SM isolated plasmid (Fig. 4A-D).

\section{The DM pEGFP plasmid has similar transfection efficiency} compared to the EndoZero and Midi kit purified plasmid The transfection efficiency of the pEGFP plasmid purified by the DM approach was compared with that of the same plasmid purified with commercial EndoZero and Quiagen Midi kits. The results presented in Fig. 5A

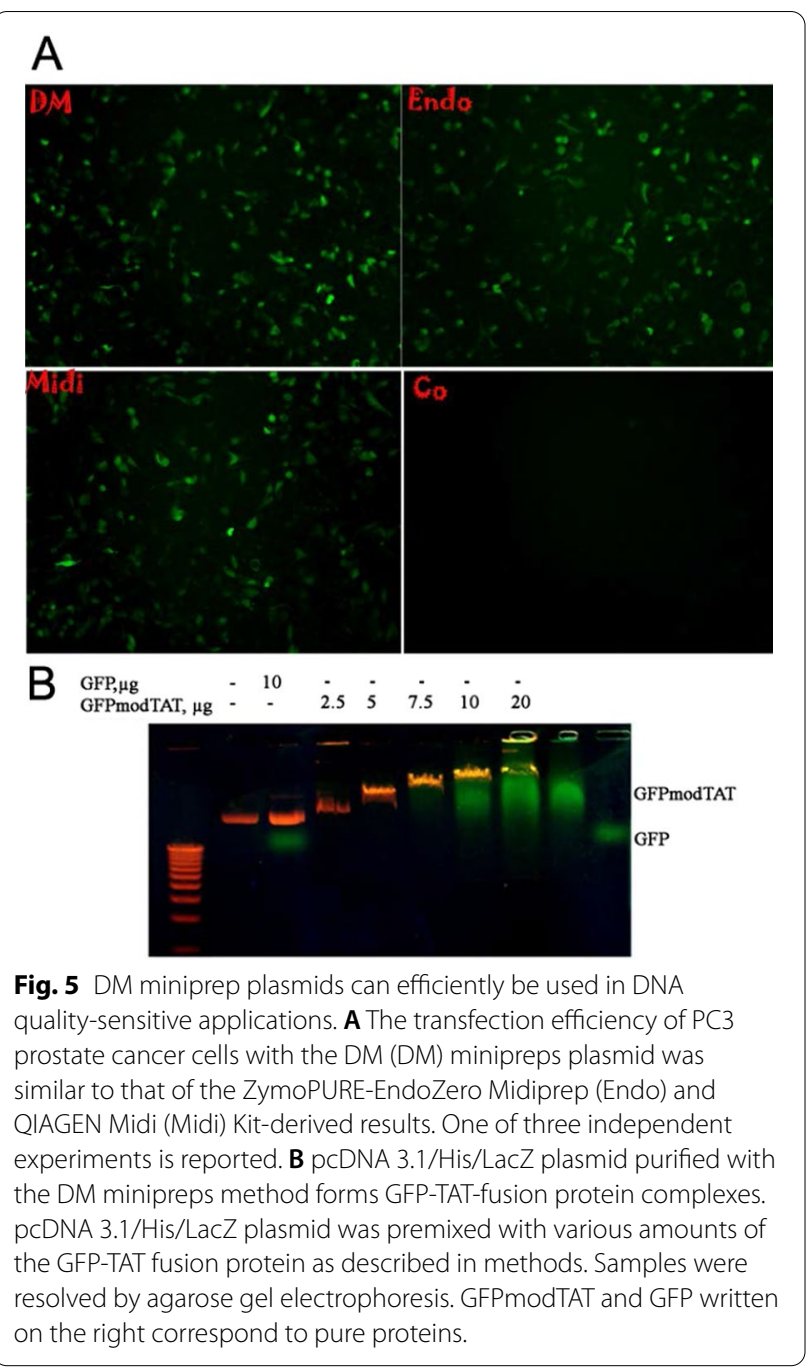

show that plasmids purified by DM miniprep were able to transfect $35 \%$ of PC3 cells, whereas EndoZero and Quiagen Midi kits purified plasmids transfected 34\% and $33 \%$ of cells, respectively. Similar transfection efficiency was observed among the three plasmids tested, suggesting that DM miniprep plasmid DNA can successfully be used in cell transfection experiments, making these experiments more cost- and time-effective.

\section{GFP-TAT binding}

To test whether the GFP-TAT fusion protein could bind to DNA in vitro, the pcDNA $3.1 / \mathrm{His} / \mathrm{LacZ}$ plasmid was mixed with various concentrations of GFP-TAT fusion. The formed complexes were analyzed by electrophoresis on an agarose gel and stained with ethidium bromide (Fig. 5B). As seen in the figure, the migration of supercoiled DNA bands is shifted with increasing amounts of GFP-TAT fusion. These results suggested that the positively charged TAT peptide might bind to plasmid DNA. 
This binding would occur between the negative charges of plasmid DNA and the positive charges of the TAT protein transduction domain of the fusion protein via electrostatic interactions.

\section{Discussion}

Although plasmid DNA purification is a well-established method in molecular biology, the technique is still developing for time-, cost saving and decreasing labor, especially upon processing multiple samples. It was found that with a decrease in the time and speed of centrifugation of the bacterial culture, the time for resuspending the bacterial sediment using a vortex device is significantly reduced (Voo and Jacobsen 1998). The plasmid DNA recovery in miniprep occurs by alkaline cell lysis followed by neutralization with an acidic high molar potassium acetate. After neutralization, the cell debris, high molecular weight chromosomal DNA and other impurities become trapped within the soft, buoyant and highly shear-sensitive gel matrix (Ciccolini et al. 1999). The separation of the plasmid-containing solution from the floating gel matrix was carried out by high-speed centrifugation. The mixing conditions and the alkaline incubation time are critical in plasmid minipreps. Many plasmid miniprep protocols indicate 5-min incubation for the lysis of bacteria that were mixed with lysis buffer by vigorously inverting the tube. Thereafter, neutralization was carried out by adding chilled neutralization buffer and inverting the tube, followed by incubation on ice for 5 min (Qiagen Plasmid Handbook 2021). During the lysis step, intracellular constituents are released within a few seconds, and the chromosomal DNA is irreversibly denatured under extreme alkaline conditions, while plasmid DNA is capable of annealing after the neutralization step. If the alkaline environment during lysis exceeds $0.15 \pm 0.03 \mathrm{M}$ ( $\mathrm{pH} 12.9 \pm 0.2)$, irreversible denaturation of the supercoiled plasmid DNA occurs (Meacle et al. 2004). Therefore, when the lysis buffer of $0.2 \mathrm{M} \mathrm{NaOH}$ range is mixed with the resuspended cells, some of the cells will experience a locally high $\mathrm{pH}$, leading to plasmid denaturation that is shown to increase with time of exposure, and as a result, denatured supercoiled plasmid DNA appears in the final eluate of SM Miniprep (Fig. 3A-C). Yu et al. (2008) showed that such alkaline denatured supercoiled DNA has a stable conformation with unregistered, topologically constrained double strands and an intrastranded secondary structure. This form is not effective in gene transfer and constitutes important impurities due to their physical and chemical similarities with the supercoiled form (Prazeres et al. 1998). It has been shown that lysis of $4 \mathrm{ml}$ E. coli suspension occurs within 30-40 s (Ciccolini et al. 1999) and that the pure supercoiled plasmid denatured rapidly in $0.2 \mathrm{M} \mathrm{NaOH}$ (Meacle et al. 2004).
Taking into account these data and our observation that the cell suspension obtained under the conditions of steps 1 and 2 (Fig. 1C) of the DM miniprep method is lysed immediately (becomes clear) after the addition of the lysis solution, we excluded the incubation time in alkali and the mixing as well and added a neutralizing solution at once. This will minimize the exposure of the plasmid to an alkaline environment, its denaturation, and, we assume, will fall off the effect of a shear in genomic DNA. Due to the change in the rheological properties of the lysate during the lysis steps and especially the neutralization step, it is difficult to achieve gentle and efficient mixing even in miniprep format with regard to shearing genomic DNA to avoid the presence of this contaminant in the low copy number plasmid obtained by the standard miniprep method (Fig. 3A). The addition of a denser $\left(\mathrm{d}=1.16 \mathrm{~g} / \mathrm{cm}^{3}\right)$ neutralizing solution settle it to the bottom of the tube, and to ensure faster renaturation of denatured plasmids, rapid intensive mixing could be a correct strategy. Therefore, we have introduced rotor oscillation-driving mixing in the miniprep procedure. With these modifications, we demonstrated that the mixing efficiency of the solution affects the plasmid quality and yield (Fig. 2). We determined that the combination of oscillation amplitude $45^{\circ}$ and oscillation frequency $400 \mathrm{rpm}$ ensures homogenous admixing of solutions during minipreps in $5 \mathrm{~s}$, yielding the amount of plasmid DNA exceeding that of the SM isolated plasmid (Fig. 2A, C, D).

Taking into consideration the hydrodynamic aspects of fluid mixing at a relatively high rotor oscillation frequency, which can lead to a force generated by the liquid due to turbulence and might have an effect on chromosomal shearing, we assessed the presence of the host genomic DNA in plasmid preparations (Fig. 4). Unexpectedly, our results show that the action of fluid hydrodynamic stresses caused by the movement of the rotor with the indicated oscillation frequency does not affect chromosomal shearing in the DM miniprep, and the content of the host genomic DNA in the final plasmid preparation is consistently lower than that in the isolated SM plasmid (Fig. 4A-D). This is consistent with the finding that efficient mixing during neutralization achieved with a Rushton turbine and agitation speed in the range of $190-1200 \mathrm{rpm}$ does not lead to contamination of the plasmid DNA with the chromosomal fraction (Chamsart et al. 2001). However, the isolated plasmid DNAs studied are mainly found in the form of OC, even with gentle mixing. It seems that prolonged incubation in alkali and a 5-min neutralization step promote the turning of CCC to the OC isomer of the plasmid. In addition, we provide evidence that the presence of host genomic DNA is observed even when 
using a plasmid isolation kit other than the miniprep format. From the data presented in Fig. 4C, D, one can conclude that a small fraction of host genomic DNA in the range of 60-150 pg per $50 \mathrm{ng}$ of plasmid, representing an impurity of plasmid preparations and detected in plasmid samples obtained by both DM, SM minipreps and commercial non-miniprep kits, using alkaline lysis of bacterial cells, arises due to the shearing of the host chromosome during alkaline lysis of cells. Improving the selective CCCDNA plasmid/chromosomal DNA binding/elution conditions to silica membranes can help avoid plasmid preparation to contain the host genomic DNA fraction.

Surprisingly, all plasmids purified by the DM method and stored at $+4^{\circ}$ for one year showed differences in the stability of CCC form plasmid DNA (Fig. 3B). If plasmids obtained by the SM and DM (oscillation amplitude $45^{\circ}$, oscillation frequency $400 \mathrm{rpm}$ ) methods retained the CCC form as predominant, then supercoiling of high copy number plasmids isolated by DM at $270^{\circ}$ and low copy number plasmids isolated at 180 and $90^{\circ}$ of mixing options was almost completely lost, turning into an OC isoform (Fig. 3B). In E. coli, the topological state of plasmid DNA is under the control of four DNA topoisomerases that are able to alter negative supercoils from the CCC form of DNA (Higgins and Vologodskii 2015). It seems that the observation of changes in the topological behavior of plasmids that we observe after long-term storage is mediated by the copurification of Topo I/Topo III of type I enzymes, which break one strand at a time, converting the supercoiled form to the OC isoform. Thus, the DM method can differentiate plasmid isolation relative to possible copurification of the CCC plasmid-Topo I/Topo III complexes and may be of interest for supercoiling studies.

Here, we have introduced the new DM miniprep method. We assume that an increase in the yield of plasmid DNA and their higher quality achieved with DM miniprep is associated with a short exposure time to an alkaline environment, faster step of neutralization of minipreps, and more efficient mixing of solutions using rotor oscillations. The plasmid DNAs purified using the DM method and the GeneJET miniprep kit are free of the dCCCDNA plasmid topological invariant and contain less genomic DNA. We expanded the field of application of DM miniprep plasmids and showed that the quality of the plasmids is high enough to be used in studies of protein/peptide-DNA binding and that plasmids purified by the DM method can be widely used in DNA methylation analysis. Our DM method can differentiate plasmid isolation relative to possible copurification of the CCC plasmid-Topo I/Topo III complexes and may be of interest for supercoiling studies. With the DM method, miniprep is a less labor procedure for plasmid miniprep with minimal manipulation and can be considered a "semiautomatic" method, allowing up to $60 \%$ timesaving when processing 12 samples compared to SM. The above approach can be especially useful for researchers, who have limited access to laboratory supplies and equipment and require a rapid method and apparatus for isolating plasmid DNA.

\section{Supplementary Information}

The online version contains supplementary material available at https://doi. org/10.1186/s13568-022-01360-7.

Additional file 1: Fig. S1. Sequencing of DM miniprepped DNA applied for mouse PPARg2 DNA methylation analysis. Fig. S2. Sequencing of DM miniprepped DNA applied for human Oct4 promoter DNA methylation analysis.

\section{Acknowledgements}

We would like to express our gratitude to Vitaly Mironov (ELMI Ltd, Latvia) for his help and support on many aspects of the device design.

\section{Authors' contributions}

DM and IM designed and constructed the device for miniprep. MB conducted the experiments and analyzed the data. MB wrote the manuscript. All authors read and approved the manuscript.

\section{Funding}

This work was supported by an internal grant from Rīga Stradinš University No. 132311.

\section{Availability of data and materials}

The data generated and analyzed during the current study are available from the corresponding author on reasonable request.

\section{Declarations}

Ethics approval and consent to participate

This article does not contain any studies with human participants or animals performed by any of the authors.

\section{Consent for publication}

Not applicable.

\section{Competing interests}

The authors declare that they have no known competing interests.

\section{Author details}

${ }^{1}$ Institute of Microbiology and Virology, Riga Stradins University, Ratsupites Str 5, Riga 1067, Latvia. ${ }^{2}$ ELMI Ltd, A Sakharova Str 8-18, Riga 1021, Latvia.

Received: 31 January 2022 Accepted: 3 February 2022

Published online: 22 February 2022

\section{References}

Altschuler M, Heddens DK, Diveley RR Jr, Kresheck GC (1994) Plasmid DNA isolation utilizing a novel nonionic detergent. Biotechniques 17:434-436 Baryshev M, Inashkina I, Salmina K, Jackson TR, Erenpreisa J (2018) DNA methylation of the Oct4A enhancers in embryonal carcinoma cells after etoposide treatment is associated with alternative splicing and altered pluripotency in reversibly senescent cells. Cell Cycle 17(3):362-366 Baryshev M, Petrov N, Ryabov V, Popov B (2022) Transient expression of inactive RB in mesenchymal stem cells impairs their adipogenic potential and 
is associated with hypermethylation of the PPARY2 promoter. Genes Dis 9(1):165-175

Birnboim HC, Doly J (1979) A rapid alkaline extraction procedure for screening recombinant plasmid DNA. Nucleic Acids Res 7:1513-1523

Chamsart S, Patel H, Hanak JA, Hitchcock AG, Nienow AW (2001) The impact of fluid-dynamic-generated stresses on chDNA and pDNA stability during alkaline cell lysis for gene therapy products. Biotechnol Bioeng 75(4):387-392

Cheng L, LiTY, Zhang Y (2004) Rapid preparation of total nucleic acids from $E$. coli for multi-purpose applications. J Biochem Mol Biol 37:351-355

Chowdhury EH, Akaike T (2005) Rapid isolation of high quality, multimeric plasmid DNA using zwitterionic detergent. J Biotechnol 119:343-347

Ciccolini LAS, Ayazi Shamlou P, Titchener-Hooker NJ, Ward JM, Dunnill P (1999) Rheological properties of chromosomal and plasmid DNA during alkaline lysis reaction. Bioprocess Eng 21:231-237

Diogo MM, Queiroz JA, Monteiro GA, Prazeres DMF (1999) Separation and analysis of plasmid denatured forms using hydrophobic interaction chromatography. Anal Biochem 275:122-124

Frerix A, Geilenkirchen P, Muller M, Kula MR, Hubbuch J (2007) Separation of genomic DNA, RNA, and open circular plasmid DNA from supercoiled plasmid DNA by combining denaturation, selective renaturation and aqueous two-phase extraction. Biotechnol Bioeng 96:57-66

He M, Wilde A, Kaderbhai MA (1990) A simple single-step procedure for small scale preparation of Escherichia coli plasmids. Nucleic Acids Res 18(6):1660

Higgins NP, Vologodskii AV (2015) Topological behavior of plasmid DNA. Microbiol Spectr. https://doi.org/10.1128/microbiolspec.PLAS-0036-2014

Jo E, Brent R, Kaderbhai MA (1991) Minipreps of Plasmid DNA. Curr Protoc Mol Biol 15(II):1.6:1.6.1-1.6.10

Kovalenko SA, Tanaka M, Ozawa T (1994) Simple methods for preparation of plasmid DNA yielding long and accurate sequence data. Nucleic Acids Res 22:5771-5772

Kuhnert P, Nicolet J, Frey J (1995) Rapid and accurate identification of Escherichia coli K-12 strains. Appl Environ Microbiol 61:4135-4139

Lezin G, Kosaka Y, Yost HJ, Kuehn MR, Brunelli L (2011) A one-step miniprep for the isolation of plasmid DNA and lambda phage particles. PLOS ONE 6(8):e23457. https://doi.org/10.1371/journal.pone.0023457

Meacle FJ, Lander R, Ayazi Shamlou P, Titchener-Hooker NJ (2004) Impact of engineering flow conditions on plasmid DNA yield and purity in chemical cell lysis operations. Biotechnol Bioeng. https://doi.org/10.1002/bit. 20114

Prazeres DMF, Schluep T, Cooney C (1998) Preparative purification of supercoiled plasmid DNA using anion exchange. J Chromatogr A 806(1):31-45

QIAGEN Plasmid Purification Handbook 02/2021

Sato M, Akasaka E, Saitoh I, Ohtsuka M, Nakamura S, Sakurai T, Watanabe S (2012) A simplified protocol for the semi-large scale recovery of plasmids from Escherichia coli grown on agar plates. J Biomed Sci Eng 5:406-408

Sato M, Inada E, Saitoh I, Matsumoto Y (2014) Microbial and enzyme technology: an efficient and convenient method for MiniPrep analysis of recombinant plasmids. J Biomed Sci Eng 7:105-107

Sousa F, Prazeres DMF, Queiroz JA (2009) Improvement of transfection efficiency by using supercoiled plasmid DNA purified with arginine affinity chromatography. J Gene Med 11:79-88

Vinograd J, Lebowitz J (1966) Physical and topological properties of circular DNA. J Gen Physiol 49:103-125

Voo KS, Jacobsen BM (1998) Rapid resuspension of pelleted bacterial cells for miniprep plasmid DNA isolation. Biotechniques 24:240-243

Yu J, Zhang Z, Cao K, Huang X (2008) Visualization of alkali-denatured supercoiled plasmid DNA by atomic force microscopy. Biochem Biophys Res Commun 374(3):415-418

\section{Publisher's Note}

Springer Nature remains neutral with regard to jurisdictional claims in published maps and institutional affiliations.

\section{Submit your manuscript to a SpringerOpen ${ }^{\circ}$ journal and benefit from:}

- Convenient online submission

- Rigorous peer review

- Open access: articles freely available online

- High visibility within the field

- Retaining the copyright to your article

Submit your next manuscript at $\boldsymbol{\nabla}$ springeropen.com 